\title{
The neural basis of auditory temporal discrimination in girls with fragile $X$ syndrome
}

\author{
Scott S. Hall • Elizabeth Walter • Elena Sherman • \\ Fumiko Hoeft • Allan L. Reiss
}

Received: 1 December 2008 /Accepted: 5 February 2009/Published online: 18 February 2009

(C) Springer Science + Business Media, LLC 2009

\begin{abstract}
Fragile X syndrome (FXS) is a common genetic disorder in which temporal processing may be impaired. To our knowledge however, no studies have examined the neural basis of temporal discrimination in individuals with FXS using functional magnetic resonance imaging (fMRI). Ten girls with fragile $\mathrm{X}$ syndrome and ten developmental age-matched typically developing controls performed an auditory temporal discrimination task in a $3 \mathrm{~T}$ scanner. Girls with FXS showed significantly greater brain activation in a left-lateralized network, comprising left medial frontal gyrus, left superior and middle temporal gyrus, left cerebellum, and left brainstem (pons), when compared to a developmental age-matched typically developing group of subjects who had similar in-scanner task performance. There were no regions that showed significantly greater brain activation in the control group compared to individuals with FXS. These data indicate that networks of brain regions involved in auditory temporal processing may be dysfunctional in FXS. In particular, it is possible that girls with FXS employ left hemispheric resources to overcompensate for relative right hemispheric dysfunction.
\end{abstract}

Keywords Fragile X syndrome - Temporal processing · Discrimination $\cdot \mathrm{fMRI}$

S. S. Hall $(\bowtie) \cdot$ E. Walter $\cdot$ E. Sherman $\cdot$ F. Hoeft $\cdot$ A. L. Reiss

Department of Psychiatry and Behavioral Sciences,

School of Medicine, Stanford University,

401 Quarry Road,

Stanford, CA 94305-5795, USA

e-mail: hallss@stanford.edu

\section{Introduction}

Fragile X Syndrome (FXS) is the most common cause of inherited mental retardation, affecting up to one in 4,000 individuals in the general population [5]. FXS most commonly occurs in association with an expansion of a CGG trinucleotide sequence within the FMR 1 gene located at band 27.3q of the X chromosome [35]. This expansion generally leads to hypermethylation of the gene, resulting in reduced production of FMR1 protein (FMRP). FMRP is thought to be involved in regulating mRNA expression by either stimulating or inhibiting protein translation at the molecular level [34]. The deficiency of FMRP observed in FXS leads to abnormalities in both brain development and function, primarily thought to be related to aberrant development and plasticity of synapses [10].

Reduced levels of FMRP are a risk factor for behavioral and cognitive deficits that encompass the areas of memory and learning, information and sensory processing, and social and emotional conduct [11, 23, 27, 28, 30]. Previous research has also indicated that individuals with FXS possess characteristic structural abnormalities in specific brain regions including increased volume of the caudate nucleus $[6,15]$. Other brain regions show reduction in size in comparison to control subjects, including the cerebellar vermis and the superior temporal gyrus [22, 29], key brain regions involved in motor control and auditory processing [17].

Several lines of data suggest that abnormal processing or modulation of sensory (particularly auditory) stimuli and motor output may contribute to neurocognitive dysfunction in FXS. For example, children with FXS often show hyperresponsivity to auditory stimuli [21], have a fast and fluctuating rate of speech [12], and demonstrate hyperactive 
and stereotyped motor behavior [11]. Recent studies of the FMR1 knockout mouse suggests that they have abnormal sensitivity to auditory stimuli [4, 25] and also show deficits of cerebellar learning that depend on precise timing (cerebellar-mediated classical eye-blink conditioning; [18]).

A fundamental component of sensorimotor function is the correct perception and organization of the temporal characteristics of sensory stimuli and motor output. Accordingly, abnormalities in timing perception could contribute to a broad array of cognitive, language and motor deficits in FXS [3, 33]. In a recent study employing magneto-encephalography (MEG), Rojas and colleagues [31] found that in patients with FXS, the amplitude for the $\mathrm{N} 100 \mathrm{~m}$ auditory evoked field was significantly greater than that of controls, suggesting that more neurons are activated by auditory stimuli in FXS. Activation was also found to be significantly less right-lateralized than in control patients, suggesting that neural networks involved in responding to auditory stimulation may be dysfunctional in individuals with FXS.

Temporal processing has been studied in healthy controls using functional magnetic resonance imaging (fMRI) by examining brain activation in response to auditory stimuli $[16,24,26]$. In the study conducted by Rao and colleagues, for example, four consecutive tones (organized into two pairs) were presented in sequence and subjects were asked to discriminate whether the interval between the second tone pair was shorter or longer than the first tone pair. Activations specific to the timing task included regions in the basal ganglia, cerebellum, thalamus, right inferior and superior parietal cortex, bilateral premotor cortex, and right dorsolateral prefrontal cortex. A right hemispheric bias was observed for this temporal processing, especially in the parietal cortex. Interestingly, a pitch discrimination task performed by the participants using similar stimuli activated a strongly left-lateralized set of regions in the frontal (dorsolateral prefrontal cortex, anterior cingulate cortex) and parietal (inferior and superior parietal) cortex.

In the current imaging study, we employed fMRI to investigate auditory temporal processing in FXS using an fMRI paradigm similar to that employed by Rao et al. Specifically, we compared brain activation in individuals with FXS to a developmental age-matched group of individuals without FXS while subjects were performing an auditory temporal discrimination task. Through this analysis, we wanted to gain an increased understanding of the neural processes underlying auditory processing in FXS. This study is of particular interest to understanding the pathogenesis of neurobehavioral abnormalities in FXS, as the regions that Rao and colleagues implicate in different components of timing perception in healthy controls include key areas that have been reported to be morphologically and/or functionally aberrant in individuals with FXS: the cerebellum, caudate nucleus, prefrontal cortex and parietal cortex $[15,20]$.

\section{Method}

Subjects

Ten female subjects diagnosed with FXS (mean age= 18.7 years, $\mathrm{SD}=3.81$ years) and ten female developmental age-matched typically developing (TD) controls with no known history of neurological or psychiatric disorder also participated in the study (mean age $=14.7$ years, $S D=$ 2.95 years). The subjects with FXS were participating in a longitudinal study investigating the development of cognition, behavior and brain function in adolescents and young adults with FXS. Two of the girls with FXS were taking antidepressant and stimulant medication and two were taking antidepressant medication only. TD subjects were recruited from the local area and were selected so that their developmental ages matched the subjects with FXS. None of the TD subjects were taking medications. All subjects were right-handed. Table 1 shows the individual subject data.

The mean IQ of individuals with FXS was 86.3 (SD= 16.41) while the mean IQ of TD participants was 110.2 (SD= 10.84). Although the mean ages and IQ's of the groups were significantly different $[t(18)=2.58, p<0.05 ; t(18)=-3.84$, $p<0.005$, respectively], the mean developmental ages $[\mathrm{MA}=\mathrm{CA} \times(\mathrm{IQ} / 100)]$ of the groups were comparable [FXS group $=15.78(\mathrm{SD}=2.82)$; TD group $=15.79(\mathrm{SD}=$ $3.32), t(18)=-0.006, p=$ n.s.]. The mean FMRP level of individuals with FXS, as measured by standard techniques [36] was $52.3 \%$ (range $=22.5 \%$ to $81.0 \%$ ). Parents of all children under 18 years consented for their child to participate, and children assented if they understood the procedure, consistent with guidelines approved by the local Institution Review Board. All participants aged over 18 years consented to take part in the study.

\section{Experimental design}

A blocked design was employed with rest (R), experimental (E) and control (C) conditions presented in the following order: R, E, C, E, C, E, C, E, C, R, with each block lasting 30 -s in duration. In each experimental and control block, instructions were displayed on the screen for $6 \mathrm{~s}$, followed by the presentation of four trials. Each trial lasted for $6 \mathrm{~s}$. Trials began with a fixation cross presented at the center of the screen for $250 \mathrm{~ms}$. A standard auditory tone was then presented for $750 \mathrm{~ms}$. After a $1,000 \mathrm{~ms}$ interval, a second 
Table 1 Characteristics of the sample and in-scanner behavioral performance

\begin{tabular}{|c|c|c|c|c|c|c|}
\hline & Age (years) & IQ & Developmental age $^{\mathrm{a}}$ & Percent correct & Mean response time (ms) & Percent FMRP \\
\hline \multicolumn{7}{|c|}{ FXS subject } \\
\hline 1 & 19.2 & 65 & 12.48 & 87.50 & 787.75 & 37 \\
\hline 2 & 19.4 & 76 & 14.74 & 81.25 & 977.00 & 27 \\
\hline 3 & 21.6 & 71 & 15.34 & 81.25 & 1048.88 & 22.5 \\
\hline 4 & 24.0 & 84 & 20.16 & 100.00 & 1033.50 & 62 \\
\hline 5 & 19.0 & 80 & 15.22 & $-{ }^{\mathrm{b}}$ & $-{ }^{\mathrm{b}}$ & 81 \\
\hline 6 & 22.8 & 84 & 19.15 & 68.75 & 835.48 & 57.5 \\
\hline 7 & 11.4 & 95 & 10.83 & 62.50 & 1014.56 & 64.5 \\
\hline 8 & 16.7 & 95 & 15.86 & 68.75 & 599.13 & 70.5 \\
\hline 9 & 18.3 & 89 & 16.29 & 100.00 & 826.75 & 39 \\
\hline 10 & 14.3 & 124 & 17.77 & 81.25 & 899.58 & 62 \\
\hline \multicolumn{7}{|c|}{ TD subject } \\
\hline 1 & 18.7 & 111 & 20.76 & 87.5 & 874.61 & $-^{\mathrm{c}}$ \\
\hline 2 & 13.9 & 107 & 14.02 & 56.25 & 878.75 & - \\
\hline 3 & 19.6 & 102 & 19.98 & 68.75 & 861.75 & - \\
\hline 4 & 14.5 & 121 & 17.53 & 68.75 & 875.59 & - \\
\hline 5 & 11.2 & 117 & 13.09 & 93.75 & 980.12 & - \\
\hline 6 & 12.3 & 119 & 14.64 & 87.5 & 1509.84 & - \\
\hline 7 & 15 & 112 & 16.78 & 56.25 & 1128.72 & - \\
\hline 8 & 15.8 & 112 & 17.71 & 100 & 1090.48 & - \\
\hline 9 & 15.8 & 84 & 13.23 & 62.5 & 994.39 & - \\
\hline 10 & 10.6 & 117 & 10.18 & 100 & 687.88 & - \\
\hline
\end{tabular}

${ }^{\mathrm{a}}$ Developmental age $=$ chronological age $\times \mathrm{IQ} / 100$

${ }^{\mathrm{b}}$ Button box malfunction

${ }^{\mathrm{c}}$ Not applicable

"comparison" auditory tone was presented. In experimental blocks, the duration of the second tone was either 250, 500, 1,000 or $1,250 \mathrm{~ms}$ (presented in pseudo-random order within each block). In control blocks, the second tone duration was the same as the first tone duration (i.e., $750 \mathrm{~ms}$ ). All tones were presented to the subject at a pitch of $1,500 \mathrm{~Hz}$ via headphones. On experimental trials, the task of the subject was to determine whether the comparison tone was longer or shorter than the standard tone during the remainder of the trial. If subjects thought the comparison tone was longer than the standard tone, they were instructed to press button 1 with their index finger. If subjects thought the comparison tone was shorter than the standard tone, they were instructed to press button 2 with their middle finger. In control blocks, subjects were instructed to press either button 1 or button 2 after the presentation of the two equal duration tones. Thus, the control blocks were identical to the experimental blocks in almost every respect except that no discrimination was required during control blocks. All subjects had received prior training on the task during a 1-h session conducted in a mock scanner. Total task time was 5 min.

\section{fMRI acquisition}

Data were collected at Stanford University Lucas Center (Stanford, CA USA) using a 3.0-T Signa scanner (General Electric, Milwaukee, WI USA) with a prototype head coil and button box. A T2*-weighted gradient echo spiral pulse sequence [9] was used with the following acquisition parameters: volumes $=645, \mathrm{TR}=2,000 \mathrm{~ms}, \mathrm{TE}=30 \mathrm{~ms}$, flip angle $=80^{\circ}, \mathrm{FOV}=200 \mathrm{~mm}$, matrix $=64 \times 64,28$ oblique slices, and resolution $=3.125 \times 3.125 \times 4.0 \mathrm{~mm}$ with a $0.5 \mathrm{~mm}$ gap.

\section{Data analysis}

Statistical analysis was performed using Statistical Parametric Mapping software (SPM2; Wellcome Department of Cognitive Neurology, London, UK). The first four functional volumes were discarded to establish equilibrium magnetization. After image reconstruction, each subject's data were analyzed for motion, and realigned to the first functional volume. Sessions were then normalized using the mean functional volume resampled to $2 \times 2 \times 2 \mathrm{~mm}$ 
voxels in Montreal Neurological Institute (MNI) stereotaxic space (12 nonlinear iterations, $7 \times 8 \times 7$ non-linear basis functions, medium regularization, sinc interpolation). Spatial smoothing was done with a Gaussian filter of $8 \mathrm{~mm}$ full-width at half-maximum. Each subject's data was high-pass filtered at $128 \mathrm{~s}$, and analyzed using a fixed-effects model.

Group analysis was performed with a random-effects model [7]. One-sample $t$-tests were conducted between experimental vs control blocks for the FXS and TD groups separately. In addition, a two-sample $t$-test was conducted by comparing activation in the FXS group and the TD group. Significant clusters of activation in all tests were determined using the joint expected probability distribution with height $(p=0.01)$ and extent thresholds $(p=0.05)$.

Since there were significant differences in age and IQ between the FXS and TD groups, we examined correlations between IQ and age, and brain activation, in order to exclude the possibility that the brain regions we found significantly different between FXS and TD groups were due to these factors. Contrast values at regions of interest (ROIs), defined as all areas that showed a significant difference in brain activation between FXS and TD groups, were extracted and correlated with age and IQ in the FXS group. Further, in order to examine whether activation of these brain regions could potentially covary with development and FMRP level, contrast values extracted from these ROIs were also correlated with developmental age and FMRP level in the FXS group.

SPMs were overlaid onto the SPM2 high-resolution $\mathrm{T} 1$ individual template for viewing. Coordinates of activation were converted from MNI to Talairach space using the mni2tal function (http://www.mrc-cbu.cam.ac. uk/Imaging/Common/mnispace.shtml). Brain regions were then identified from these $\mathrm{x}, \mathrm{y}$ and $\mathrm{z}$ coordinates using the Talairach Daemon (Research Imaging Center, University of Texas Health Science Center in San Antonio (RIC UTHSCSA, TX, USA) and confirmed with the Talairach atlas [32].

\section{Results}

In-scanner behavioral data are shown in Fig. 1. A 2 (group) $\times 4$ (condition) repeated measures analysis of variance (ANOVA) indicated that there was no significant difference between the groups in the percentage of correct responses obtained [FXS group $=81.25 \%(\mathrm{SD}=13.26)$; TD group $=78.13 \% \quad(\mathrm{SD}=17.49), \quad[F(1,17)=0.19, p=$ n.s. $]$. There were also no significant difference between the groups in overall response times [FXS group $=891.40 \mathrm{~ms}$ $(\mathrm{SD}=146.21)$; TD group=971.49 $\mathrm{ms}(\mathrm{SD}=237.57),[F(1$, 17) $=0.76, p=$ n.s.]. There was no significant effect of condition for percentage correct responding $[F(3,51)=$ $0.33, p=$ n.s.], though there was a significant main effect of condition for the response time data $[F(3,51)=4.20, p=$ $0.01]$. Post-hoc analyses revealed that response times were significantly longer for the 750 vs $250 \mathrm{~ms}$ discrimination than for the 750 vs $500 \mathrm{~ms}$ discrimination $(p<0.05)$.

Brain activation during the control task was subtracted from the activation in the experimental task. Figure 2 shows brain activation for the single group analyses, with the group of FXS participants shown in red and the TD group in green. In the TD group, activation was primarily observed in the left lingual gyrus of the occipital lobe (BA 18), as well as in the left inferior parietal cortex (BA 40). Sites that were close to reaching the cut-off threshold were found in the right caudate head and right cerebellum. In the FXS group, activation was seen across a much more widely distributed set of brain regions, including the left precentral gyrus (BA 6), left middle frontal gyrus (BA 6/ 10), bilateral cingulate gyrus (BA 23/32), and bilateral activation in superior temporal gyrus (BA 22/38) which extended superiorly into left inferior parietal cortex, as well as in left cerebellum and bilateral caudate body (see Table 2). Thus, activation in the FXS group was more diffuse and distributed over a larger number of regions.

Figure 3 shows brain activation following subtraction of the TD group activation from the FXS group activation during the task. After subtraction, greater activation in the
Fig. 1 Mean percent correct (left panel) and mean response time (right panel) for FXS and TD groups as a function of comparison tone duration (250, $500,1,000$ or $1,250 \mathrm{~ms})$. The standard tone duration $(750 \mathrm{~ms})$ is represented by the vertical line
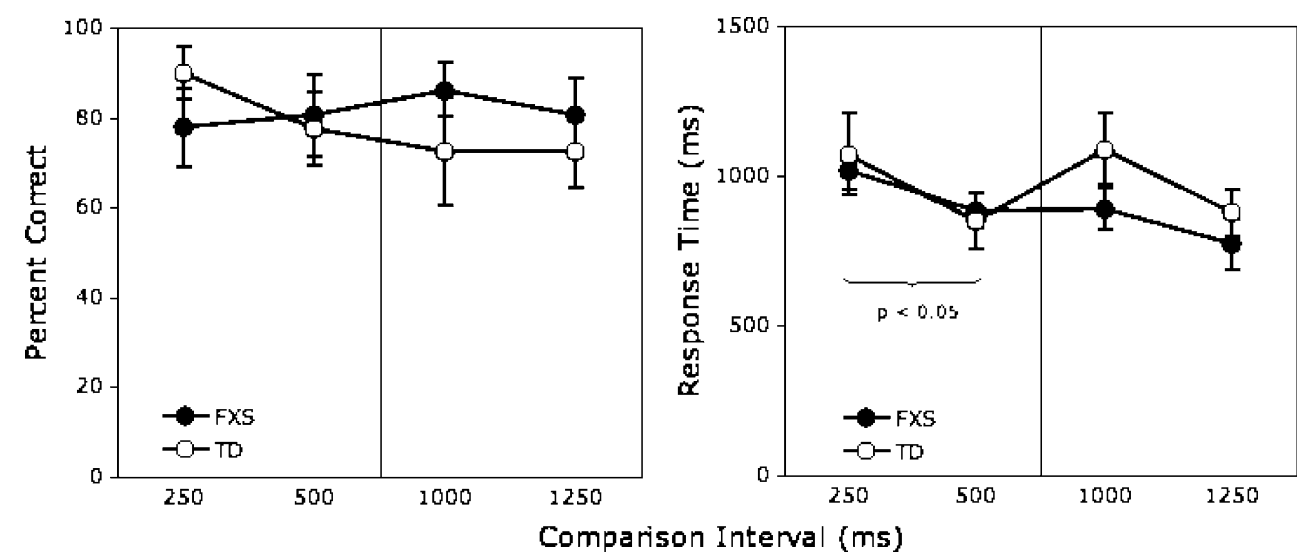


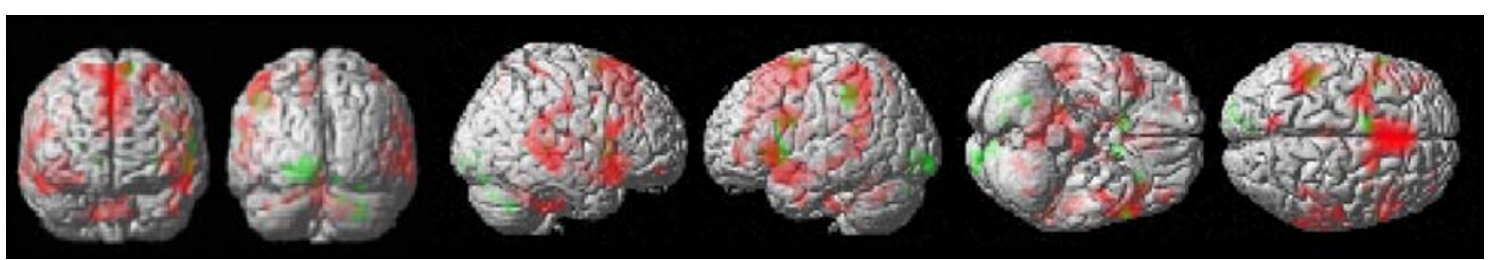

Fig. 2 Experimental minus control task contrasts for the FXS group (activation shown in red) and TD group (activation shown in green). ( $p<$ 0.01 , uncorrected)

FXS group was observed over a number of brain regions including the left medial frontal gyrus (BA 6), left middle temporal gyrus (BA 21), left cerebellum (anterior lobe) and left brainstem (pons; see Fig. 3 and Table 3). On the other hand, there were no regions that showed significantly greater activation in the TD group compared to FXS.

Since the FXS group was significantly older and had lower IQ's compared to the TD group, we performed confirmatory analyses to exclude the possibility that these factors contributed to brain activation differences observed between FXS and the TD group. If the greater activation observed in the FXS group compared to the TD group is independent of age and IQ, then one might not expect to observe increases in brain activation with older age or lower IQ in the FXS group. As expected, contrast values of significantly activated brain regions showed no significant correlation with age or IQ.

We also speculated that if the regions that showed significantly greater activation in FXS compared to the controls were playing a compensatory role that changed with development, these regions may show increasingly greater brain activation with greater developmental age in FXS. However, only the left pons showed a significant (positive) correlation with mental age in the FXS group $\left(r^{2}=0.53, p=\right.$ 0.03). Further supporting the specific role of these regions and its association with development in FXS, there were no significant correlations between mental age and brain activation in any region in the TD group. Interestingly, performance (percent correct) did correlate with activation in the cerebellum in the TD group $\left(r^{2}=0.53, p=0.02\right)$, and performance was significantly correlated with activation in middle temporal gyrus in the FXS group $\left(r^{2}=0.45, p=0.05\right)$. In the FXS group, contrast values of significantly activated regions showed no significant correlation with FMRP level.

\section{Discussion}

The main goal of our study was to examine the neural basis of auditory time perception in girls with FXS by comparing

Table 2 Experimental minus control task contrasts for FXS and TD groups

\begin{tabular}{|c|c|c|c|c|c|c|c|c|}
\hline & \multirow[t]{2}{*}{ Anatomical description } & \multirow[t]{2}{*}{ BA } & \multicolumn{3}{|c|}{ Peak Location } & \multirow[t]{2}{*}{ No. of voxels } & \multirow[t]{2}{*}{$Z$-max } & \multirow{2}{*}{$\begin{array}{l}\text { Cluster } p \text {-value } \\
\text { (uncorrected) }\end{array}$} \\
\hline & & & $x$ & $y$ & $z$ & & & \\
\hline \multicolumn{9}{|l|}{ FXS } \\
\hline Frontal & Left precentral gyrus & 6 & -36 & -1 & 26 & 570 & 3.33 & 0.003 \\
\hline Frontal & Right precentral gyrus & 44 & 65 & 14 & 9 & 418 & 3.15 & 0.009 \\
\hline Frontal & Left middle frontal gyrus & 6 & -40 & 6 & 48 & 775 & 3.32 & 0.001 \\
\hline Limbic & Left cingulate gyrus & 23 & -8 & -24 & 29 & 260 & 3.13 & 0.033 \\
\hline Limbic & Right cingulate gyrus & 32 & 6 & 29 & 32 & 3983 & 4.62 & $<0.0005$ \\
\hline Temporal & $\begin{array}{l}\text { Left superior temporal gyrus, } \\
\text { extending to inferior parietal cortex }\end{array}$ & 38 & -50 & 17 & -18 & 3329 & 3.86 & $<0.0005$ \\
\hline Temporal & Right superior temporal gyrus & 38 & 44 & 19 & -18 & 1079 & 3.57 & $<0.0005$ \\
\hline \multirow[t]{4}{*}{ Temporal } & Right superior temporal gyrus & 22 & 48 & -29 & 0 & 1906 & 3.37 & $<0.0005$ \\
\hline & Left cerebellum & & -2 & -47 & -11 & 1544 & 4.45 & $<0.0005$ \\
\hline & Left caudate body & & -16 & -1 & 18 & 300 & 2.86 & 0.023 \\
\hline & Right caudate body & & 10 & -1 & 17 & 380 & 3.33 & 0.012 \\
\hline \multicolumn{9}{|l|}{ TD } \\
\hline Parietal & Left inferior parietal & 40 & -44 & -33 & 40 & 322 & 3.07 & 0.037 \\
\hline \multirow[t]{3}{*}{ Occipital } & Left lingual gyrus & 18 & -10 & -83 & 1 & 428 & 3.26 & 0.019 \\
\hline & Right cerebellum & & 28 & -60 & -32 & 149 & 3.69 & 0.139 \\
\hline & Right caudate head & & 10 & 12 & -2 & 240 & 3.8 & 0.066 \\
\hline
\end{tabular}




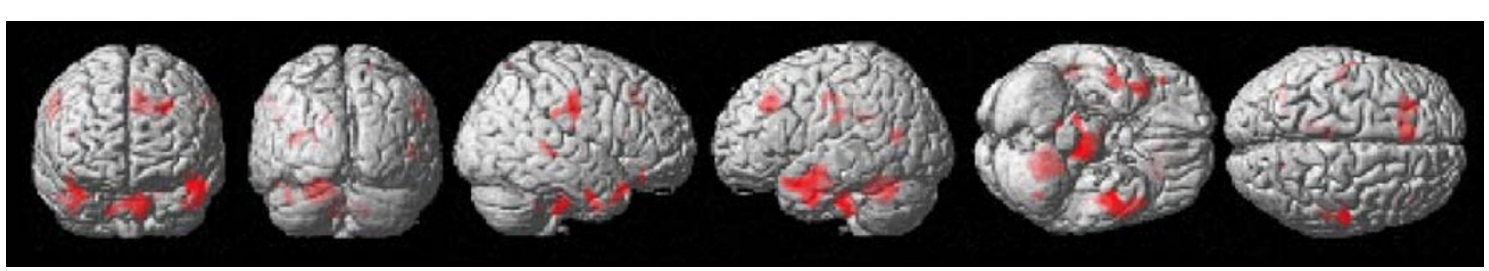

Fig. 3 Brain regions that showed significantly greater activation in FXS compared to the TD group in the experimental $\gg$ control contrast. $(p<$ 0.01 , uncorrected)

them with healthy individuals matched for in-scanner task performance and mental age. We found that several areas including a left-lateralized network of frontal, temporal and cerebellar regions displayed greater activation in the FXS group in comparison to the TD group. This finding could be explained by the possibility that FMRP, the protein product of the FMRI gene, plays a critical role in the development of brain regions underlying auditory processing.

Previous fMRI studies related to temporal processing in healthy controls have reported increased activation in several brain regions. Rao and colleagues [26] reported activation in the basal ganglia, cerebellum, bilateral premotor, right dorsolateral prefrontal cortex, right inferior parietal, right superior temporal and left middle temporal gyrus during a time duration discrimination task similar to the one employed in our study. Nenadic and colleagues [24] found activation in the right dorsolateral and middle prefrontal cortices, as well as left anterior cingulate, bilateral temporal (superior and middle temporal gyri) and bilateral basal ganglia (caudate nucleus and putamen) when subtracting a control task from an auditory timing task. Similarly, Harrington and colleagues [13] asked participants to perform a temporal judgment, but analyzed the beginning of the trial (devoted to encoding) separately from the later portions of the trial (presumably reflecting decision-making and response-planning). During the encoding portion, these researchers observed activation in regions of frontal cortex (bilateral medial frontal, left anterior cingulate, right posterior cingulate, and left superior frontal gyrus), temporal cortex (right parahippocampal gyrus and left superior temporal gyrus), and parietal cortex (bilateral inferior and superior parietal), in addition to activations in right lingual gyrus in occipital cortex, bilateral cerebellum and bilateral caudate nucleus. They found a bilateral, though primarily left-lateralized, group of activations in frontal (superior, middle and inferior frontal gyri), temporal (superior temporal), and parietal (supramarginal gyrus and superior parietal) cortex to be involved specifically in the decisionmaking portion of the trials. In searching for commonalities in the temporal discrimination literature, it should be noted that, while often bilateral, the reported activity is generally right-lateralized during the timing task itself, perhaps indicating a right hemispheric bias for temporal processing.

In the current study, individuals in the TD group demonstrated activation in the left lingual gyrus (BA 18) as well as in the left inferior parietal cortex (in addition to near-threshold activity in the right caudate head and right cerebellum). One reason why we did not observe significant activation in other brain regions that have been implicated in auditory time perception studies (e.g., [26]) may be due to the simple and relatively easy in-scanner task we adopted. The methods employed by Rao et al. [26] and Harrington et al. [13] involved the use of two sets of brief tone pairs, demarcating two time intervals, which subjects were then asked to compare. In order to study temporal processing in a population of developmentally delayed participants, we designed a task such that FXS and developmental age-matched TD individuals could equally and successfully perform the time discrimination task in the scanner. Because of the relative difficulty of discriminating between four tones (and the two intervals defined by them), we chose to adopt a simpler task involving the discrimination of two tones, with one tone always played for $750 \mathrm{~ms}$ to serve as a reference. Participants were asked to determine whether the second tone was played for a longer or shorter period than the reference tone. In our task therefore, the

Table 3 Between group contrasts of experimental vs control conditions

\begin{tabular}{|c|c|c|c|c|c|c|c|c|}
\hline \multirow[t]{2}{*}{$\mathrm{FXS} \gg \mathrm{TD}$} & \multirow[t]{2}{*}{ Anatomical description } & \multirow[t]{2}{*}{$\mathrm{BA}$} & \multicolumn{3}{|c|}{ Peak location } & \multirow[t]{2}{*}{ No. of voxels } & \multirow[t]{2}{*}{ Z-max } & \multirow{2}{*}{$\begin{array}{l}\text { Cluster } p \text {-value } \\
\text { (uncorrected) }\end{array}$} \\
\hline & & & $x$ & $y$ & $z$ & & & \\
\hline Frontal & Left medial frontal gyrus & 6 & -6 & 29 & 37 & 500 & 3.22 & 0.035 \\
\hline \multirow[t]{3}{*}{ Temporal } & Left superior and middle temporal gyrus & $21 / 38$ & -44 & -5 & -15 & 829 & 3.72 & 0.009 \\
\hline & Left cerebellum & & -20 & -53 & -19 & 843 & 3.49 & 0.009 \\
\hline & Left pons & & -4 & -25 & -27 & 450 & 3.72 & 0.044 \\
\hline
\end{tabular}


working memory component was quite reduced (two items, as opposed to four) in order to reduce the difficulty and thus ensure participant success. Our behavioral results indicate that the two groups were matched for in-scanner task performance, which allowed us to conduct a proper comparison between the two groups.

As a result of making our task easier in order to equate behavioral performance, we may have inadvertently reduced the cognitive load required to perform this relatively simple task, leading to reduced activations in the TD individuals. It seems that a wide set of brain areas was still recruited in the FXS group, perhaps as a result of the relative difficulty they experience in processing auditory stimuli in a complex auditory environment such as an MRI scanner. Indeed, in the group analysis of FXS participants, significant activation occurred in many regions, including left middle frontal and bilateral cingulate gyrus. It is possible that these frontal regions are involved because of an increase in task difficulty for the FXS group relative to the TD group. Previous research has described increased activation in this region during more difficult temporal discrimination tasks and it has been proposed that this occurs as a result of the greater role that working memory plays in more challenging temporal discrimination tasks [13].

More interestingly, a direct comparison between FXS and the TD group revealed several brain areas with significant activation differences that have been implicated in auditory time perception; however, these areas were localized to contralateral left homologous regions. The most notable findings were regions that showed significantly greater activation in FXS compared to the TD individuals: in the medial frontal gyrus, superior and middle temporal gyrus, cerebellum and pons, with relative activation differences predominantly in the left hemisphere. In comparison, Nenadic and colleagues [24] found activations in right middle and inferior frontal gyrus as well as left anterior cingulate, and bilateral superior and middle temporal, and bilateral basal ganglia during a time discrimination task. Their sites of activation in frontal and temporal cortex are reasonably similar to those in the FXS group, with the notable difference that our FXS participants tend to activate left- rather than right-lateralized regions of frontal cortex.

The left inferior parietal cortex was activated in both of our groups when analyzed separately (in the FXS group it is reported as part of a larger cluster weighted toward the superior temporal gyrus). This region has been implicated in time perception studies [14], and may be involved in intrahemispheric networks used for timing, though there is also some evidence that its role is in the decision-making process, rather than the timing encoding per se [13]. Interestingly, other studies have implicated right inferior parietal cortex [19] in a time estimation task, as well as in a task requiring determination of a sound's location in azimuth [1, 2]. In addition, Rao and colleagues [26] found the right inferior parietal cortex to be activated during an fMRI study similar to our own and proposed that this region may be responsible for the regulation of pulses generated by an internal clock. Rao also suggests that the inferior parietal cortex may be involved in maintaining attention, as attention-keeping processes are central to the subjective perception of the passage of time. It is not yet clear why our participants recruited left inferior parietal cortex rather than the expected right inferior parietal cortex.

Greater activation was observed in the left cerebellum in FXS, a region that has been suggested to be involved in decision-making processes of temporal discrimination tasks, specifically by optimizing sensory input from auditory nuclei and thus allowing the comparison of different time intervals in one's working memory [26]. An alternative explanation is that the primary function of the cerebellum is to assist in the process of encoding timing intervals [13]. It is important to note that in previous studies, activation has been found in the cerebellar vermis, a region noted for its cognitive functions related to both tactile perception and working memory. It is possible that the cerebellar vermis monitors and adjusts sensory input from the cerebral cortex. Activation differences were also found in the left basal ganglia. Activation in the caudate nucleus and the putamen $[24,26]$ has been observed at the beginning of trial epochs, thus suggesting that the basal ganglia may participate in encoding time intervals and forming one's representation of time.

Our findings are limited because only a small number of subjects were included in the study, thus severely limiting the generalizability of our results. In addition, four of the girls with FXS were taking antidepressant medication at the time of the study that may have affected the degree to which we found activations in these subjects. Subjects were also matched for developmental age, but not for chronological age, introducing the possibility that younger participants may recruit different neural networks for temporal processing than older subjects as a result of agebased neurodevelopmental differences. Thus, group brain activation may have varied because of the difference in absolute age between our groups of subjects. Our correlation analysis, however, did not support this possibility; there were no significant correlations between age and brain activation in any of the regions that differed between the two groups. Future research should focus on examining temporal processing in FXS in larger groups of chronological and developmental-age matched individuals to control for the development of brain circuitry and its consequent implications for regional activation during temporal processing tasks. In addition, it may be fruitful to investigate 
other aspects of auditory stimuli such as pitch perception, or the processing of speech sounds. Finally, event-related designs could help us further disentangle the different roles of the brain regions discussed here during temporal processing.

In conclusion, we found that brain activation during a temporal auditory discrimination task in girls with FXS differed significantly from typically developing individuals. Activation in girls with FXS occurred diffusely over several brain regions, while activation in the TD group was seen in localized regions, mainly in the left inferior parietal cortex and left lingual gyrus. While these findings should be considered preliminary due to the small sample sizes, these data support a previous MEG study in which brain activation in response to auditory stimulation was found to be more diffuse and less right-lateralized in patients with FXS than in controls [31]. These findings may indicate that FMRP plays a critical role in the development of brain circuitry involved in auditory temporal processing in FXS. Given that synaptic pruning is impaired in FXS, particularly in occipital and temporal cortex [8], it seems likely that an overabundance of synapses in these regions could create "noise" in the processing of the auditory signal. Deficits in temporal processing may be responsible for the "cluttered" speech and motor coordination problems shown by individuals with FXS, particularly in males. These individuals could potentially benefit from early training on temporal discrimination tasks. Future studies should look more comprehensively at primary auditory processing in FXS, perhaps using a tonotopic fMRI approach.

Acknowledgements This research was supported by NIH grants MH50047 and MH01142.

\section{References}

1. Alain $\mathrm{C}, \mathrm{He} \mathrm{Y}$, Grady C. The contribution of inferior parietal lobe to auditory spatial working memory. J Cogn Neurosci. 2008;20:28595.

2. Arnott S, Grady C, Hevenor SJ, Graham S, Alain C. The functional organization of auditory working memory as revealed by fMRI. J Cogn Neurosci. 2005;17:819-31.

3. Artieda J, Pastor MA, Lacruz F, Obeso JA. Temporal discrimination is abnormal in Parkinson's disease. Brain 1992;115:199210.

4. Chen L, Toth M. Fragile X mice develop sensory hyperreactivity to auditory stimuli. Neuroscience 2001;103:1043-50.

5. Crawford DC, Meadows KL, Newman JL, Taft LF, Pettay DL, Gold LB, et al. Prevalence and phenotype consequence of FRAXA and FRAXE alleles in a large, ethnically diverse, special education-needs population. Am J Med Genet. 1999;64:495-507.

6. Eliez S, Blasey CM, Freund LS, Hastie T, Reiss AL. Brain anatomy, gender and IQ in children and adolescents with fragile $\mathrm{X}$ syndrome. Brain 2001;124:1610-8.
7. Friston KJ, Holmes AP, Price CJ, Buchel C, Worsley KJ. Multisubject fMRI studies and conjunction analyses. Neuroimage 1999;10:385-96.

8. Galvez R, Greenough WT. Sequence of abnormal dendritic spine development in primary somatosensory cortex of a mouse model of the fragile X mental retardation syndrome. Am J Med Genet. 2005;135A:155-60.

9. Glover GH, Lemieux SK, Drangova M, Pauly JM. Decomposition of inflow and blood oxygen level-dependent (BOLD) effects with dual-echo spiral gradient-recalled echo (GRE) fMRI. Magn Reson Med. 1996;35:299-308.

10. Greenough WT, Klintsova AY, Irwin SA, Galvez R, Bates KE, Weiler IJ. Synaptic regulation of protein synthesis and the fragile X protein. Proc Natl Acad Sci USA. 2001;98:7101-6.

11. Hall S, DeBernardis G, Reiss A. Social escape behaviors in children with fragile $\mathrm{X}$ syndrome. J Autism Dev Disord. 2006;36:935-47.

12. Hanson DM, Jackson AW, Hagerman RJ. Speech disturbances (cluttering) in mildly impaired males with the Martin-Bell/fragile X syndrome. Am J Med Genet. 1986;23:195-206.

13. Harrington DL, Boyd LA, Mayer AR, et al. Neural representation of interval encoding and decision making. Cogn Brain Res. 2004;21:193-205.

14. Harrington DL, Haaland KY, Knight RT. Cortical networks underlying mechanisms of time perception. J Neurosci. 1998;18:108595.

15. Hessl D, Rivera SM, Reiss AL. The neuroanatomy and neuroendocrinology of fragile x syndrome. Ment Retard Dev Disabil Res Rev. 2004;10:17-24.

16. Hinton SC, Harrington DL, Binder JR, Durgerian S, Rao SM. Neural systems supporting timing and chronometric counting: an FMRI study. Brain Res Cogn Brain Res. 2004;21:183-92.

17. Ivry RB, Keele SW, Diener HC. Dissociation of the lateral and medial cerebellum in movement timing and movement execution. Exp Brain Res. 1988;73:167-80.

18. Koekkoek SK, Yamaguchi K, Milojkovic BA, Dortland BR, Ruigrok THJ, Maex R, et al. Deletion of FMR1 in Purkinje cells enhances parallel fiber LTD, enlarges spines, and attenuates cerebellar eyelid conditioning in Fragile $\mathrm{X}$ syndrome. Neuron 2005;47:339-52.

19. Macar F, Lejeune H, Bonnet M, Ferrara A, Pouthas V, Vidal F, et al. Activation of the supplementary motor area and of attentional networks during temporal processing. Exp Brain Res. 2002; 142:475-85.

20. Menon V, Kwon H, Eliez S, Taylor AK, Reiss AL. Functional brain activation during cognition is related to FMR1 gene expression. Brain Res. 2000;877:367-70.

21. Miller LJ, McIntosh DN, McGrath J, et al. Electrodermal responses to sensory stimuli in individuals with fragile $\mathrm{X}$ syndrome. Am J Med Genet. 1999;83:268-79.

22. Mostofsky SH, Mazzocco MM, Aakulu G, Warsofsky IS, Denckla MB, Reiss AL. Decreased cerebellar posterior vermis size in fragile X syndrome: correlation with neurocognitive performance. Neurology 1998;50:121-30.

23. Munir F, Cornish KM, Wilding J. Nature of the working memory deficit in fragile-X syndrome. Brain Cogn. 2000; 44:387-401.

24. Nenadic I, Gaser C, Volz H, Rammsayer T, Hager F, Sauer H. Processing of temporal infomation and the basal ganglia: new evidence from fMRI. Exp Brain Res. 2003;148:238-46.

25. Nielsen DM, Derber WJ, McClellen DA, Crnic LS. Alterations in the auditory startle response in Fmrl targeted mutant mouse models of fragile X syndrome. Brain Res. 2002;927:8-17.

26. Rao SM, Mayer AR, Harrington DL. The evolution of brain activation during temporal processing. Nat Neurosci. 2001;4:31723. 
27. Reiss AL, Freund L. Behavioral phenotype of fragile X syndrome: DSM-III-R autistic behavior in male children. Am J Med Genet. 1992;43:35-46.

28. Reiss AL, Freund LS, Baumgardner TL, Abrams MT, Denckla MB. Contribution of the FMR1 gene mutation to human intellectual dysfunction. Nat Genet. 1995;11:331-4.

29. Reiss AL, Lee J, Freund L. Neuroanatomy of fragile X syndrome: the temporal lobe. Neurology 1994;44:1317-24.

30. Riddle JE, Cheema A, Sobesky WE, Gardner SC, Taylor AK, Pennington BF, Hagerman RJ. Phenotypic involvement in females with the FMR1 gene mutation. Am J Ment Retard. 1998;102:590-601.

31. Rojas DC, Benkers TL, Rogers SJ, Teale PD, Reite ML, Hagerman RJ. Auditory evoked magnetic fields in adults with fragile X syndrome. Neuroreport 2001;12:2573-6.

32. Talairach J, Tournoux P. Co-planar stereotaxic atlas of the human brain. New York: Thieme; 1988.
33. Tallal P, Miller SL, Bedi G, Byma G, Wang X, Nagarajan SS, et al. Language comprehension in language-learning impaired children improved with acoustically modified speech. Science 1996;271(5245):81-4.

34. Tassone F, Hagerman RJ, Ikle DN, Dyer PN, Lampe M, Willemsen R, et al. FMRP expression as a potential prognostic indicator in fragile X syndrome. Am J Med Genet. 1999;84:250 61.

35. Verkerk AJ, Pieretti M, Sutcliffe JS, Fu YH, Kuhl DP, Pizzuti A, et al. Identification of a gene (FMR-1) containing a CGG repeat coincident with a breakpoint cluster region exhibiting length variation in fragile X syndrome. Cell 1991;65:905-14.

36. Willemsen R, Smits A, Mohkamsing S, van Beerendonk H, de Haan A, de Vries B, et al. Rapid antibody test for diagnosing fragile $\mathrm{X}$ syndrome: a validation of the technique. Hum Genet 1997;99:308-11. 